\title{
Non-axisymmetric instability of axisymmetric magnetic fields
}

\author{
A. Bonanno ${ }^{1,2}$ and V. Urpin ${ }^{1,3}$ \\ 1 INAF, Osservatorio Astrofisico di Catania, Via S.Sofia 78, 95123 Catania, Italy \\ e-mail: abo@oact.inaf.it \\ 2 INFN, Sezione di Catania, Via S.Sofia 72, 95123 Catania, Italy \\ 3 A.F.Ioffe Institute of Physics and Technology and Isaac Newton Institute of Chile, Branch in St. Petersburg, 194021 St. Petersburg, \\ Russia
}

Received 22 January 2008 / Accepted 10 April 2008

\section{ABSTRACT}

\begin{abstract}
Context. The MHD instabilities can generate complex field topologies even if the initial field configuration is a very simple one. Aims. We consider the stability properties of magnetic configurations containing a toroidal and an axial field. In this paper, we concentrate mainly on the behavior of non-axisymmetric perturbations in axisymmetric magnetic configurations.

Methods. The stability is treated by a linear analysis of ideal MHD equations.

Results. In the presence of an axial field, it is shown that the instability can occur for a wide range of the azimuthal wavenumber $m$, and its growth rate increases with increasing $m$. At given $m$, the growth rate is at its maximum for perturbations with the axial wave-vector that makes the Alfvén frequency approximately vanishing. We argue that the instability of magnetic configurations in the ideal MHD can typically be dominated by perturbations with very short azimuthal and axial wavelengths.
\end{abstract}

Key words. magnetohydrodynamics (MHD) - instabilities - stars: magnetic fields

\section{Introduction}

A wide variety of MHD instabilities can occur in magnetized astrophysical bodies where they play an important role in the evolution and formation of various structures and in enhancing transport processes, among others. The onset of instabilities can be caused both by hydrodynamic motions (for instance, differential rotation) or properties of the magnetic configuration. Even magnetic fields with a relatively simple topology (for example, a purely toroidal field) can be subject to instability. Magnetic fields generated by the dynamo action or stretched by hydrodynamic motions are topologically more complex and can cause this sort of instability as well. Which field strength and topology can sustain a stable magnetic configuration is still rather uncertain despite all the extensive work already done (see Borra et al. 1982; Mestel 1999 for review).

The simplest and best-studied magnetic configuration is most likely a purely toroidal one. This has been known since the paper by Tayler (1957), where stability properties of the toroidal field $B_{\varphi}$ are determined by the parameter $\alpha=\operatorname{dln} B_{\varphi} / \operatorname{dln} s$ where $s$ is the cylindrical radius. The field is unstable to axisymmetric perturbations if $\alpha>1$ and to non-axisymmetric perturbations if $\alpha>-1 / 2$. The growth time of instability is close to the time taken for an Alfvén wave to travel around the star on a toroidal field line. Numerical modeling by Braithwaite (2006) confirms that the toroidal field with $B_{\varphi} \propto s$ or $\propto s^{2}$ is unstable to the $m=1$ mode ( $m$ is the azimuthal wave number) as predicted by Tayler $(1957,1973)$. However, even a purely toroidal field can be stable in the region where it decreases rapidly with $s$. A purely toroidal field cannot be stable through the whole star because the stability condition for axisymmetric modes $(\alpha<1)$ is incompatible with the condition that the electric current in the $z$ direction has no singularity at $s \rightarrow 0$, which implies $\alpha>1$. The stability of the toroidal field in rotating stars has been considered by
Kitchatinov \& Rüdiger (2007), who argue that the magnetic instability is essentially three-dimensional and that the finite thermal conductivity creates a strong destabilizing effect. Terquem \& Papaloizou (1996) and Papaloizou \& Terquem (1997) considered the stability of an accretion disk with the toroidal magnetic field and found that the disks containing a purely toroidal field are always unstable and calculated the spectra of unstable modes in the local approximation.

The stability properties of purely poloidal magnetic fields are also well-studied. It has been understood since the papers by Wright (1973) and Markey \& Tayler $(1973,1974)$ that the poloidal field is subject to dynamical instabilities in the neighborhood of points (or lines) where the poloidal field is vanishing (neutral points/lines). These authors recognized first that the magnetic field in the neighborhood of a neutral line resembles that of a toroidal, pinched discharge, which is known to be unstable. The instability of a poloidal field is also rather fast: its growth time can reach a crossing time of few Alfvén times (Van Assche et al. 1982; Braithwaite \& Spruit 2006) that is very short, for example, compared to the time-scales of stellar evolution. However, the instability of a poloidal field can be suppressed by the addition of a toroidal field in the neighborhood of neutral points (Markey \& Tayler 1973; Wright 1973).

Conversely, the addition of even a relatively weak poloidal field alters the stability properties of the toroidal field substantially. For example, if the poloidal field is uniform and relatively weak, the instability condition of axisymmetric modes reads $\alpha>-1$, at variance with the condition of instability for a purely toroidal field (see, e.g., Knobloch 1992; Dubrulle \& Knobloch 1993), which predicts that an unstable toroidal field configuration has $\alpha>-1 / 2$. Therefore, a weak poloidal field has a destabilizing effect. However, a strong enough poloidal field can suppress the instability of the toroidal field. It turns out that configurations containing comparable toroidal and poloidal 
fields are more stable than purely toroidal or purely poloidal ones (Prendergast 1956; Tayler 1980) and, generally, the possibility exists that there are configurations containing mixed fields, which have no instabilities arising on a dynamical time-scale. In his study of unstable magnetic configurations Tayler (1980) has not found any instability if the axial field $B_{z}>0.3 B_{\varphi}$ for instance, even though such configurations can be unstable for a wide range of the azimuthal wavenumber $m$ if $B_{z}$ is weaker. With numerical simulations Braithwaite \& Nordlund (2006) studied the stability of a random initial field in the stellar radiative zone and found that the stable magnetic configurations generally have the form of tori with comparable poloidal and toroidal field strengths.

In this paper, we consider in detail the stability properties of magnetic configurations containing the toroidal and axial magnetic fields with respect to non-axisymmetric perturbations. We show that the instability may occur for a wide range of the azimuthal wavenumber $m$, and the growth rate is typically higher for higher $m$. Unstable modes with large $m$ have a very short vertical lengthscale, so it can be hard to resolve them in numerical calculations. Depending on the profile $B_{\varphi}(s)$ and the ratio $B_{z} / B_{\varphi}$, the instability can occur in two regimes that have substantially different growth rates.

The remainder of this paper is arranged as follows. In Sect. 2, we derive the equation that governs the eigenfunctions and eigenvalues of the magnetic field. We describe the numerical procedure and present the results of calculations in Sect. 3. A brief discussion of the results is given in Sect. 4.

\section{Basic equations}

Let us consider the stability of an axisymmetric cylindrical magnetic configuration in a high conductivity limit. We work in cylindrical coordinates $(s, \varphi, z)$ with the unit vectors $\left(\boldsymbol{e}_{s}, \boldsymbol{e}_{\varphi}, \boldsymbol{e}_{z}\right)$. We assume that the azimuthal field depends on the cylindrical radius alone, $B_{\varphi}=B_{\varphi}(s)$, but the axial magnetic field $B_{z}$ is constant.

In the incompressible limit, the MHD equations read

$$
\begin{aligned}
& \frac{\partial \boldsymbol{v}}{\partial t}+(\boldsymbol{v} \cdot \nabla) \boldsymbol{v}=-\frac{\nabla p}{\rho}+\frac{1}{4 \pi \rho}(\nabla \times \boldsymbol{B}) \times \boldsymbol{B}, \\
& \nabla \cdot \boldsymbol{v}=0, \\
& \frac{\partial \boldsymbol{B}}{\partial t}-\nabla \times(\boldsymbol{v} \times \boldsymbol{B})=0, \\
& \nabla \cdot \boldsymbol{B}=0 .
\end{aligned}
$$

In the basic state, the gas is assumed to be in hydrostatic equilibrium, then

$$
\frac{\nabla p}{\rho}=\frac{1}{4 \pi \rho}(\nabla \times \boldsymbol{B}) \times \boldsymbol{B} .
$$

In this paper, we consider the stability of non-axisymmetric perturbations. Since the basic state is stationary and axisymmetric, the dependence of perturbations on $t, \varphi$, and $z$ can be taken in the form $\exp \left(\sigma t-i k_{z} z-i m \varphi\right)$ where $k_{z}$ is the wave-vector in the axial direction and $m$ the azimuthal wavenumber. In stellar conditions, such a local analysis in the z-direction applies if $k_{z}$ satisfies the inequality $k_{z} s>1$ (we assume that the lengthscale in the axial direction is $\sim s$ in stars). Small perturbations will be indicated by subscript 1 , while unperturbed quantities will have no subscript. Then, the linearized Eqs. (1)-(4) are

$\sigma \boldsymbol{v}_{1}=-\frac{\nabla p_{1}}{\rho}+\frac{1}{4 \pi \rho}\left[\left(\nabla \times \boldsymbol{B}_{1}\right) \times \boldsymbol{B}+(\nabla \times \boldsymbol{B}) \times \boldsymbol{B}_{1}\right]$, $\nabla \cdot v_{1}=0$

$\sigma \boldsymbol{B}_{1}-(\boldsymbol{B} \cdot \nabla) \boldsymbol{v}_{1}+\left(\boldsymbol{v}_{1} \cdot \nabla\right) \boldsymbol{B}=0$

$\nabla \cdot \boldsymbol{B}_{1}=0$

Eliminating all variables in favor of the radial velocity perturbation $v_{1 s}$, we obtain

$\frac{\mathrm{d}}{\mathrm{d} s}\left[\frac{1}{\lambda}\left(\sigma^{2}+\omega_{A}^{2}\right)\left(\frac{\mathrm{d} v_{1 s}}{\mathrm{~d} s}+\frac{v_{1 s}}{s}\right)\right]-k_{z}^{2}\left(\sigma^{2}+\omega_{A}^{2}\right) v_{1 s}$
$-2 \omega_{B}\left[k_{z}^{2} \omega_{B}(1-\alpha)-\frac{m(1+\lambda)}{s^{2} \lambda^{2}}\left(1-\frac{\alpha \lambda}{1+\lambda}\right)\left(\omega_{A z}+2 m \omega_{B}\right)\right.$
$\left.-\frac{m \omega_{A z}}{s^{2} \lambda^{2}}\right] v_{1 s}+\frac{4 k_{z}^{2} \omega_{A}^{2} \omega_{B}^{2}}{\lambda\left(\sigma^{2}+\omega_{A}^{2}\right)} v_{1 s}=0$,

where

$\omega_{A}=\frac{1}{\sqrt{4 \pi \rho}}\left(k_{z} B_{z}+\frac{m}{s} B_{\varphi}\right), \quad \omega_{A z}=\frac{k_{z} B_{z}}{\sqrt{4 \pi \rho}}$,

$\omega_{B}=\frac{B_{\varphi}}{s \sqrt{4 \pi \rho}}, \quad \alpha=\frac{\partial \ln B_{\varphi}}{\partial \ln s}, \quad \lambda=1+\frac{m^{2}}{s^{2} k_{z}^{2}}$.

For axisymmetric perturbations $(m=0)$, Eq. (10) recovers Eq. (11) of the paper by Bonanno \& Urpin (2007), if one assumes $B_{z}=$ const. The equations derived by Acheson (1973) and Knobloch (1992) for axisymmetric perturbations can also be recovered from Eq. (10). With appropriate boundary conditions, Eq. (10) allows the eigenvalues $\sigma$ to be determined.

Our analysis does not include gravity, which is important in stars, so the stabilizing effect of stratification is neglected. In the case of magnetic instabilities, this is justified if the work done by a perturbation against gravity is less than the energy released from the magnetic field. The corresponding condition for stellar radiative zones has been considered by Spruit (1999) (see Eq. (44)) and reads in our notations as $k_{z} s>N / \omega_{B}$, where $N$ is the buoyancy frequency. Assuming that the characteristic value of $N$ in stars is $\sim 10^{-3} \mathrm{~s}^{-1}$ and introducing the axial wavelength $\lambda_{z}=2 \pi / k_{z}$, we obtain that the effect of stratification can be neglected for perturbations with

$\lambda_{z}<\lambda_{c}=2 \times 10^{8} B_{\varphi 3} \rho_{-4}^{-1 / 2} \mathrm{~cm}$,

where $B_{\varphi 3}=B_{\varphi} / 10^{3} \mathrm{G}$ and $\rho_{-4}=\rho / 10^{-4} \mathrm{~g} / \mathrm{cm}^{3}$.

Magnetic perturbations can also be affected by the presence of dissipation, which is not considered in our analysis. Usually, magnetic diffusion is weak in stellar conditions, and its influence is unimportant if the dissipation rate that is $\sim \eta k_{z}^{2}$ is small compared to $\omega_{B}$ when $\eta \sim 10^{3} \mathrm{~cm}^{2} / \mathrm{s}$ the magnetic diffusivity. This condition yields

$\lambda_{z}>\lambda_{d}=3 \times 10^{5} B_{\varphi 3}^{-1 / 2} s_{11}^{1 / 2} \rho_{-4}^{-1 / 4} \mathrm{~cm}$,

where $s_{11}=s / 10^{11} \mathrm{~cm}$. Inequalities (11) and (12) set a range of axial wavelengths where our consideration is valid.

\section{Numerical results}

We assume that the dependence of the azimuthal magnetic field on $s$ is given by

$B_{\varphi}=B_{\varphi 0}\left(\frac{s}{s_{1}}\right)^{\alpha}$ 
where $B_{\varphi 0}$ is the field strength at $s=s_{1}$. To calculate the growth rate of the instability, it is convenient to introduce dimensionless quantities

$x=\frac{s}{s_{1}}, \quad q=k_{z} s_{1}, \quad \Gamma=\frac{\sigma}{\omega_{B 0}}, \quad \omega_{B 0}=\frac{B_{\varphi 0}}{s_{1} \sqrt{4 \pi \rho}}, \quad \varepsilon=\frac{B_{z}}{B_{\varphi 0}}$.

Then, Eq. (10) transforms into

$$
\begin{aligned}
& \frac{\mathrm{d}}{\mathrm{d} x}\left(\frac{\mathrm{d} v_{1 s}}{\mathrm{~d} x}+\frac{v_{1 s}}{x}\right)+\left(\frac{\mathrm{d} v_{1 s}}{\mathrm{~d} x}+\frac{v_{1 s}}{x}\right) \frac{\mathrm{d} \ln \Delta}{\mathrm{d} x}-q^{2}\left(1+\frac{m^{2}}{q^{2} x^{2}}\right) v_{1 s} \\
& -\frac{2 q^{2} x^{\alpha-1}}{\Gamma^{2}+f^{2}}\left\{\left[\left(1-\frac{m^{2}}{q^{2} x^{2}}\right) x^{\alpha-1}-\frac{m \varepsilon}{q x^{2}}\right](1-\alpha)-\frac{2 m f}{m^{2}+q^{2} x^{2}}\right\} v_{1 s} \\
& +\frac{4 q^{2} f^{2} x^{2(\alpha-1)}}{\left(\Gamma^{2}+f^{2}\right)^{2}} v_{1 s}=0
\end{aligned}
$$

where

$f=q \varepsilon+m x^{\alpha-1}, \quad \Delta=\frac{q^{2} x^{2}\left(\Gamma^{2}+f^{2}\right)}{m^{2}+q^{2} x^{2}}$.

Solving the eigenvalue problem numerically, we assume that the inner and outer boundaries correspond to $x_{1}=1$ and $x_{2}=2$, respectively, where we suppose $v_{1 s}\left(x_{1}\right)=v_{1 s}\left(x_{2}\right)=0$. Note, however, that the results are not qualitatively sensitive to the choice of boundaries and boundary conditions. We checked that the qualitative behavior of the growth rate does not change if the outer boundary $x_{2}$ is set to higher values. As far as the inner boundary is concerned, our approach does not allow the eigenvalues to be calculated in the case $s_{1}=0$, but we verified that low but finite values of $s_{1}$ did not lead to qualitative difference in the behavior of the growth rate, and therefore we believe that our conclusions can be extended to the case $s_{1}=0$.

Equation (15), together with the given boundary conditions at the extrema, is a two-point boundary value problem that can be solved by using the "shooting" method (Press et al. 1992). To solve Eq. (15), we used a fifth-order Runge-Kutta integrator embedded in a globally convergent Newton-Rawson iterator. We have checked that the eigenvalue was always the fundamental one, as the corresponding eigenfunction had no zero except that at the boundaries. To test the numerical procedure, we consider first the case of a purely toroidal field, $\varepsilon=0$. Stability properties of such field have been well studied since the paper by Tayler (1973), who argued that the dominant unstable mode is the one with $m=1$. The instability of the toroidal field occurs if $\mathrm{d}\left(s B_{\varphi}^{2}\right) / \mathrm{d} s>0$ or, in other words, if $B_{\varphi}$ increases with the cylindrical radius or decreases not faster than $s^{-1 / 2}$. In Fig. 1, we plot the growth rate as a function of the vertical wave-vector for $B_{z}=0$ and different values of $\alpha$. In agreement with the conclusion by Tayler (1973), $\alpha=-1 / 2$ is indeed the critical value distinguishing between stable $(\alpha<-1 / 2)$ and unstable $(\alpha>-1 / 2)$ toroidal magnetic fields. The growth rate of instability can be rather high, $\sigma \sim \omega_{B 0}$. Note that the growth rate increases with increasing $q$, and perturbations with short axial wavelengths turn out to grow the most rapidly.

In Fig. 2, we plot the dependence of $\Gamma^{2}$ on $q$ for the same $m=1$ mode but in the presence of a relatively weak axial field, $B_{z}=0.1 B_{\varphi 0}$. The addition of even very weak $B_{z}$ changes the stability properties qualitatively even though the energy contained in the axial field is very low compared to that of the toroidal field $(\sim 1 \%)$. We stress that the presence of an axial field breaks symmetry $q \rightarrow-q$. The physical reason for this is fairly simple. The Lorentz force plays a crucial role in the behavior of

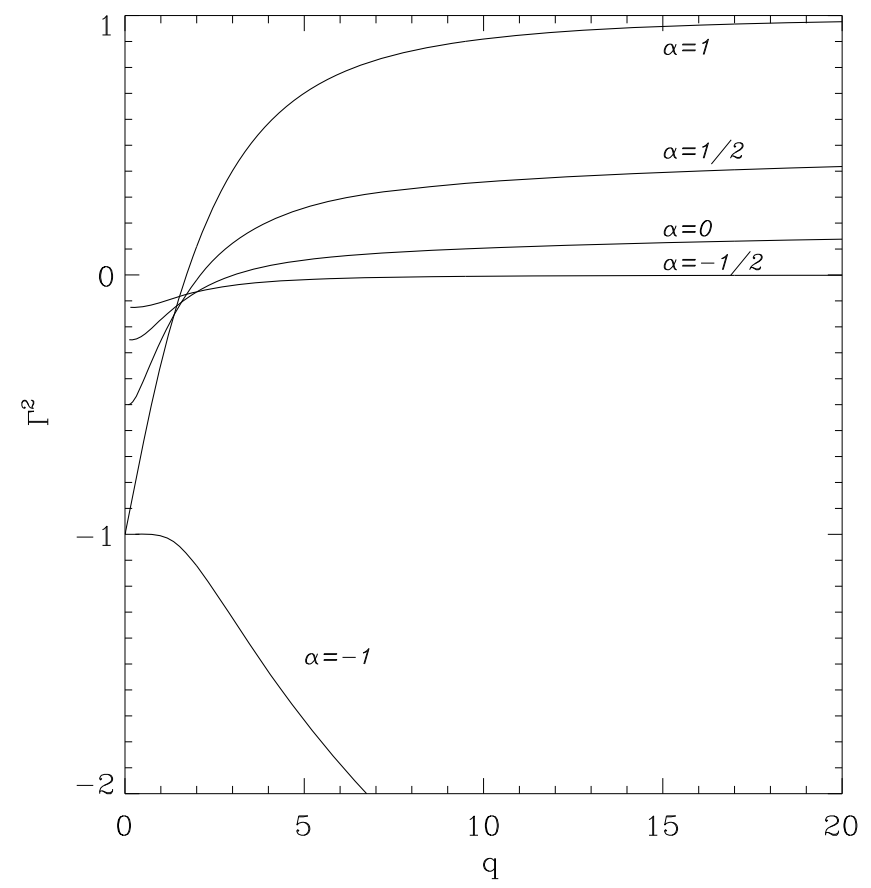

Fig. 1. The dependence of $\Gamma^{2}$ on $q$ for $B_{z}=0$ and $m=1$. Numbers near the curves indicate the values of parameter $\alpha$.

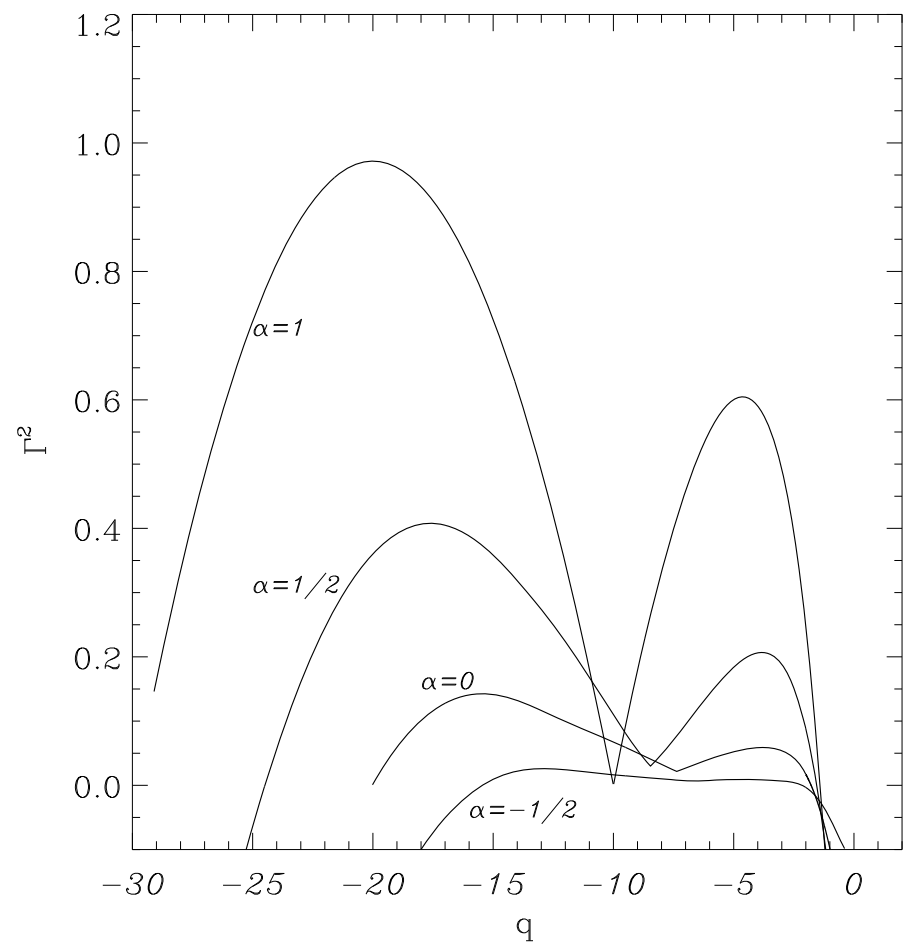

Fig. 2. The dependence of $\Gamma^{2}$ on $q$ for $\varepsilon=B_{z} / B_{\varphi 0}=0.1$ and $m=1$. Numbers near the curves correspond to values of $\alpha$.

perturbations, and this force contains a component that is proportional to the cross production of a current flowing in the basic state $\boldsymbol{j} \propto \nabla \times \boldsymbol{B}$ and the magnetic field of perturbations (see the last term on the left hand side of Eq. (6)). If $B_{z} \neq 0$, then magnetic perturbations are determined partly by the axial gradient of velocity perturbations since $\mathrm{d} \boldsymbol{B}_{1} / \mathrm{d} t=(\boldsymbol{B} \nabla) \boldsymbol{v}_{1}$, and this contribution has a different sign for positive and negative $k_{z}$. Therefore, the stability properties turn out to be dependent on the direction of an axial wavevector. However, Eq. (15) still contains 


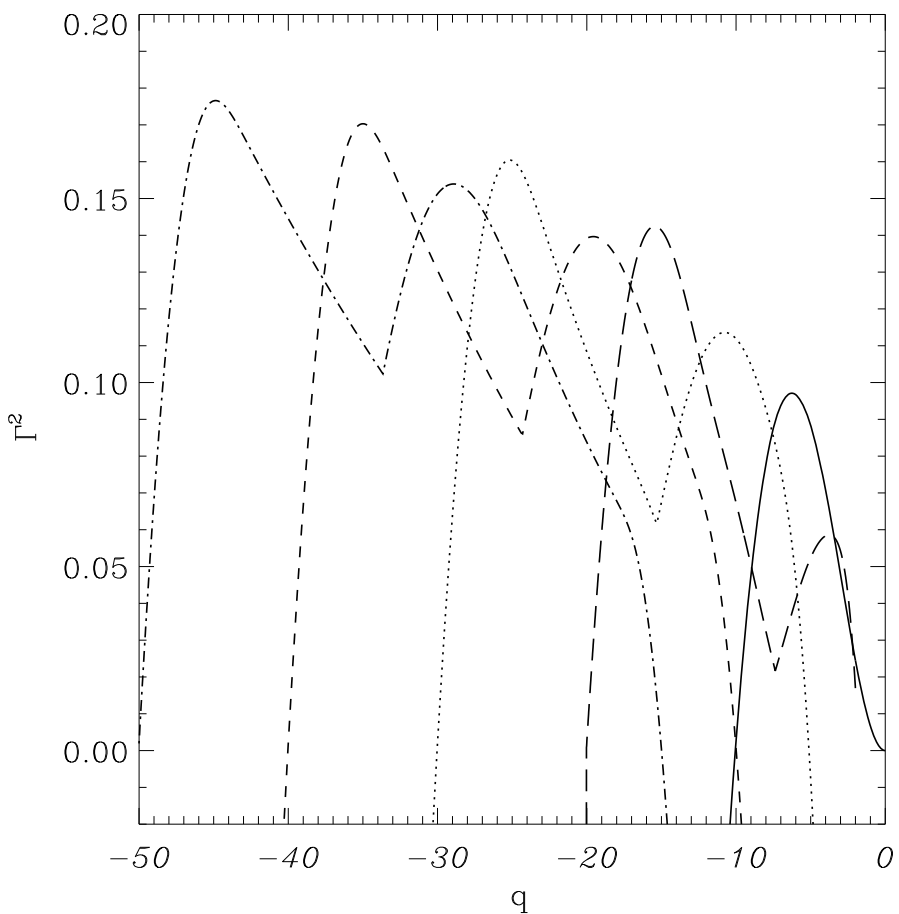

Fig. 3. The dependence of $\Gamma^{2}$ on $q$ for $\varepsilon=B_{z} / B_{\varphi 0}=0.1, \alpha=0$, and different values of $m$. Solid, long-dashed, dotted, short-dashed, and dot-dashed lines are for $m=0,1,2,3,4$, respectively.

some degeneracy because the replacements $(m, q) \rightarrow(-m,-q)$ or $(m, \varepsilon) \rightarrow(-m,-\varepsilon)$ do not change its shape. The instability occurs only for a restricted range of negative $q,-(20-30)<q<-2$ depending on the value of $\alpha$, and do not appear for positive $q$. The growth rate has two clear maxima with the higher maximum corresponding to $q \sim-1 / \varepsilon$. By the order of magnitude, the axial wave-vector of the most rapidly growing perturbation can be estimated from the condition

$\omega_{A}=\frac{1}{\sqrt{4 \pi \rho}}\left(k_{z} B_{z}+\frac{m}{s} B_{\varphi}\right) \approx 0$.

Indeed, this equation in dimensionless units reads $\omega_{A} \propto q \varepsilon+$ $m x^{\alpha-1} \approx 0$. Since $x \sim 1$ in our calculations, the condition $\omega_{A}=0$ corresponds to

$q \sim-m / \varepsilon$.

Therefore, we have $q \sim-1 / \varepsilon$ for the $m=1$ mode. The most rapidly growing modes turns out to be highly anisotropic if the axial field is weak compared to the toroidal one. Their axial wavelength $\lambda_{z}=2 \pi / k_{z} \sim 2 \pi \varepsilon s$ is much shorter than the radial and azimuthal lengthscale. The presence of an axial field shifts the threshold of unstable values of $\alpha$ : a purely toroidal field is unstable only if $\alpha>-1 / 2$, but our calculations show that the profile of $B_{\varphi}$ with $\alpha=-1 / 2$ is unstable. However, the growth rate in the case $\alpha \leq-1 / 2$ is rather low and is close to $\omega_{A z}$ rather than $\omega_{B 0}$. The growth rate grows rapidly with increasing $\alpha$ : the maximum value of $\Gamma^{2}$ is of the order of unity for $\alpha=1$ and $\sim 8$ for $\alpha=2$ (is not shown in Fig. 2).

The dependence of $\Gamma^{2}$ on $q$ in the presence of a weak axial field $(\varepsilon=0.1)$ and for different values of $m$ is shown in Fig. 3 . In this figure, $\Gamma^{2}$ is calculated for $\alpha=0$, such that the magnetic configuration is unstable even in the absence of the axial field. It turns out that this profile of $B_{\varphi}$ is unstable if $B_{z} \neq 0$, as well, but the properties of instability are substantially different. In contrast to the case of a purely toroidal field with $\alpha=0$ where only the

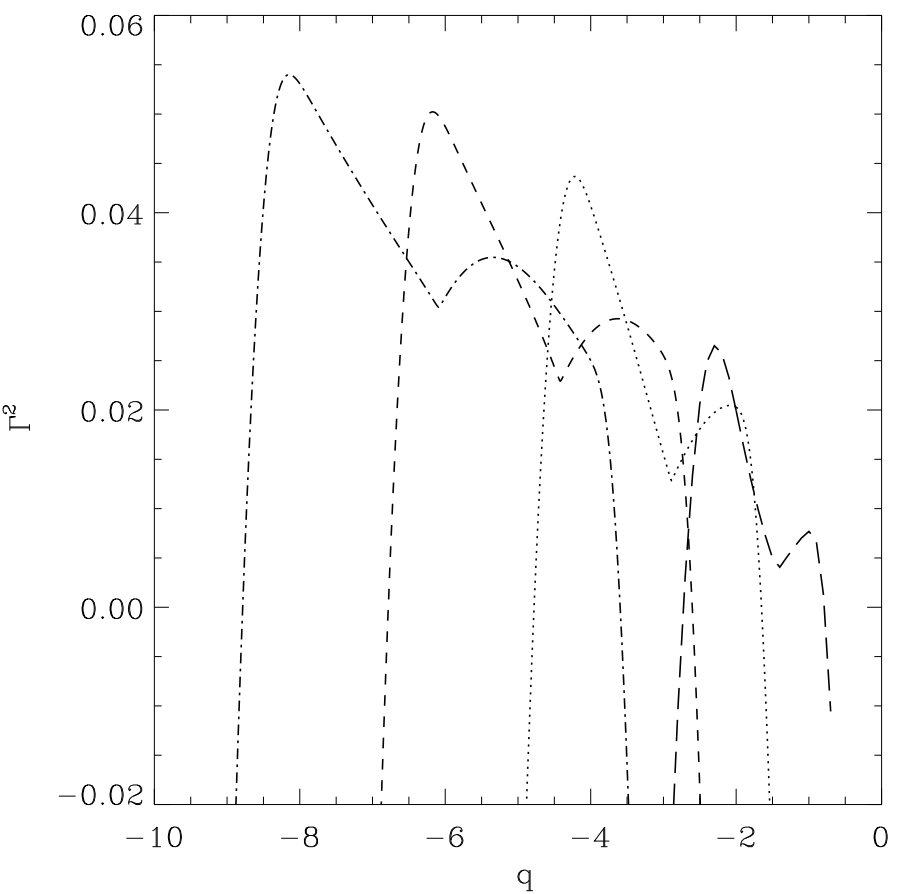

Fig. 4. The same as in Fig. 3 but for $B_{z} / B_{\varphi 0}=0.5$.

$m=1$ mode can arise (Tayler 1973), the instability occurs for modes with a wide range of $m$ including $m=0$. For $m \neq 0$, the growth rate reaches its maximum approximately at $q \approx-m / \varepsilon$, which is equivalent to the resonant condition $\omega_{A} \approx 0$. The maximum growth rate increases with an increase in $m$. Therefore, the instability of such magnetic configurations is probably dominated by the modes with large $m$ and extremely short axial wavelengths $\lambda_{z} \approx-2 \pi \varepsilon s / m$. This can cause difficulties in numerical simulations, because very high resolution in the axial and azimuthal direction would be needed to resolve the most unstable modes. Moreover a nonlinear interaction of these modes can produce axial lengthscales that are even shorter than those predicted by the linear theory.

An increase in the axial field makes the magnetic configuration more stable. In Fig. 4, we plot the growth rate for $\varepsilon=0.5$ and the same values of other parameter as in Fig. 3. The instability grows more slowly in higher $B_{z}$ : an increase in $B_{z}$ by a factor 5 leads to the decrease in $\Gamma^{2}$ approximately by a factor 2 . Nevertheless, the instability can still occur for this $\varepsilon$, and it is still efficient because its growth rate is $\sim 0.3 \omega_{B 0}$. Like the previous case, the modes with a wide range of $m$ can be unstable, and the growth rate increases with $m$. The axisymmetric mode $(m=0)$ is stable in this case (see Bonanno \& Urpin 2007). The critical value $\varepsilon$ that suppresses the instability is clearly dependent on the geometry of the basic state. For example, by using energy considerations, Tayler (1980) has not found any instability for $\varepsilon \geq 0.3$ in the configuration where the magnetic surfaces of the poloidal field are coaxial tori. On the other hand, for values of $\varepsilon$ significantly less than 0.3 , the author found instability for a wide range of values of $m$.

To illustrate the dependence of $\Gamma$ on the profile of the toroidal field, we show the growth rate as a function of $q$ for $\alpha=1$ in Fig. 5. This profile seems to be particularly interesting for astrophysical applications since the toroidal field is $\propto s$ in a neighborhood of the axis of symmetry. In the absence of the axial field, the profile with $\alpha=1$ corresponds to the toroidal field that is marginally stable to axisymmetric perturbations $(m=0)$. 


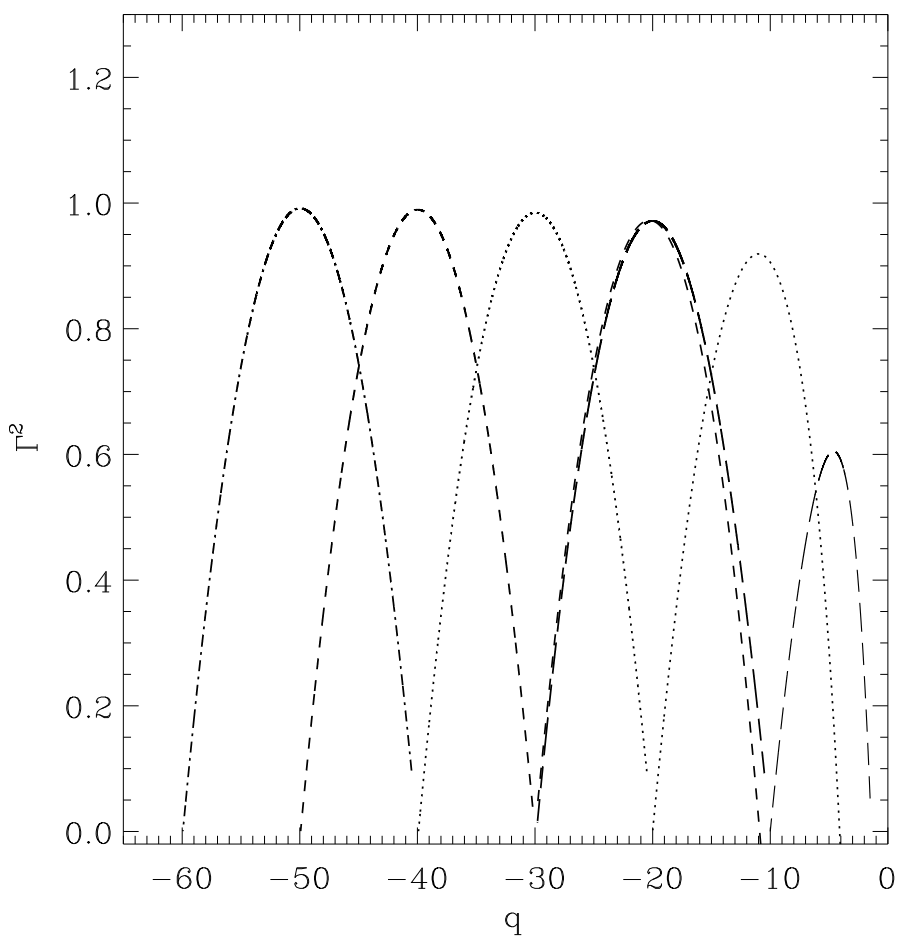

Fig. 5. The same as in Fig. 3 but for $\alpha=1$.

If $B_{z} \neq 0$, the threshold of instability can change substantially as argued by Bonanno \& Urpin (2007) and, indeed, perturbations with $m=0$ turn out to be unstable in this case. However, the non-axisymmetric perturbations grow faster and are likely to dominate the instability. The maximum growth rate is very high for them and comparable to the Alfvén frequency for the toroidal field, $\omega_{B 0}$. Remarkably, the maximum growth rate changes rather slowly with $m$ for all modes with $m \geq 2$. As in the previous cases, the maximum of $\Gamma$ is located at $q \sim-m / \varepsilon$.

In Fig. 6, we show $\Gamma^{2}$ for the case of a rapid increase in $B_{\varphi}$ with $s, B_{\varphi} \propto s^{2}$ or $\alpha=2$. The growth rate of instability is substantially higher for such $\alpha$ and can reach the value $\sim 2-3$ toroidal Alfvén frequencies. A qualitative behavior of $\Gamma$ remains same: the maximum growth rate is higher for modes with higher $m$, and these maxima correspond to very high values of the wave-vector $q \sim-m / \varepsilon$. The maximum growth rate of the axisymmetric mode $(m=0)$ is comparable to that of non-axisymmetric ones (see Bonanno \& Urpin 2007). Note that our calculations show some trend in the growth rate to reach saturation for large $m$.

Figure 7 plots the growth rate for $\alpha=-0.5$. In accordance with Tayler (1973), such a profile of $B_{\varphi}$ should be marginally stable if the axial field is vanishing. However, the presence of $B_{z}$ makes the toroidal field unstable with such $\alpha$ even if the axial field is relatively weak. The growth rate is not high in this case and is determined by the Alfvén frequency for the axial field. As usual, the maximum growth rate is higher for higher $m$, and these maxima are reached for very large $q$, which corresponds to a short axial wavelength. A comparison between Figs. 6 and 7 illustrates the difference well between two regimes of the instability first noted by Bonanno \& Urpin (2007). If $\alpha<\alpha_{\mathrm{c}}$ where $\alpha_{\mathrm{c}} \sim 1$ is some characteristic value that generally depends on $B_{z}$, the instability is relatively weak and grows on the Alfvén timescale characterized by the axial field. In contrast, if $\alpha>\alpha_{\mathrm{c}}$, the instability is much more efficient, and the growth time is of the order of the Alfvén timescale for the toroidal field.

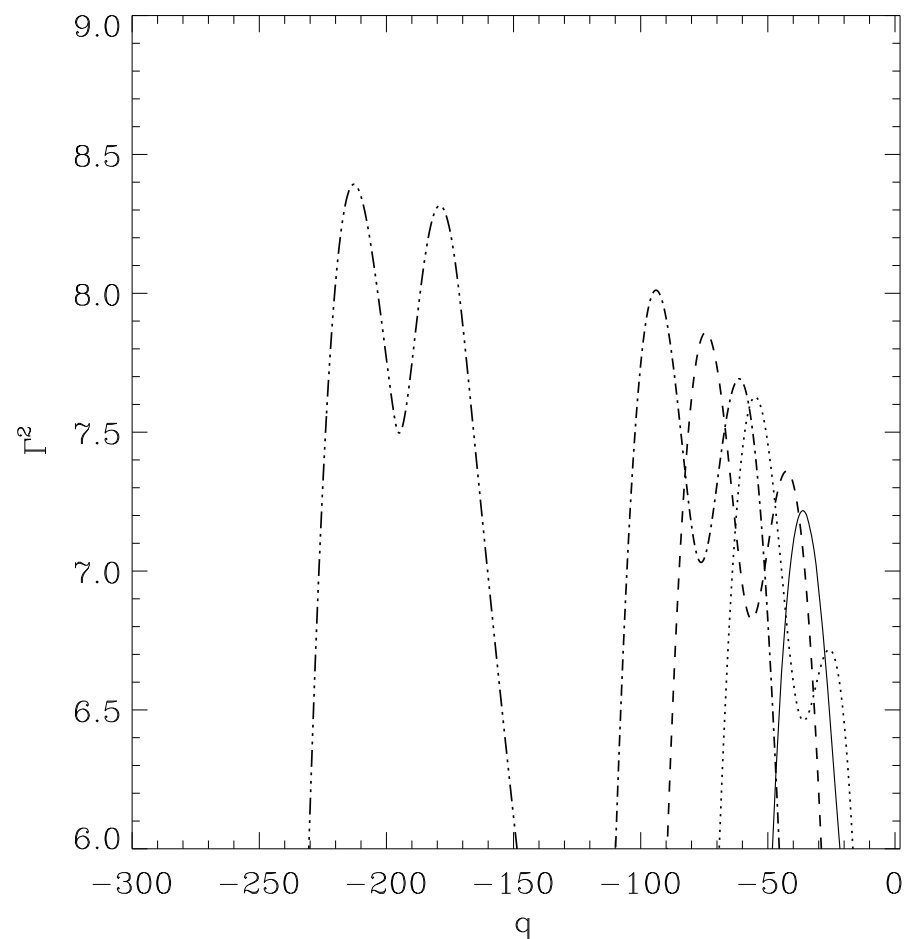

Fig. 6. The same as in Fig. 3 but for $\alpha=2$. The dash-dot-dotted line represents $m=10$.

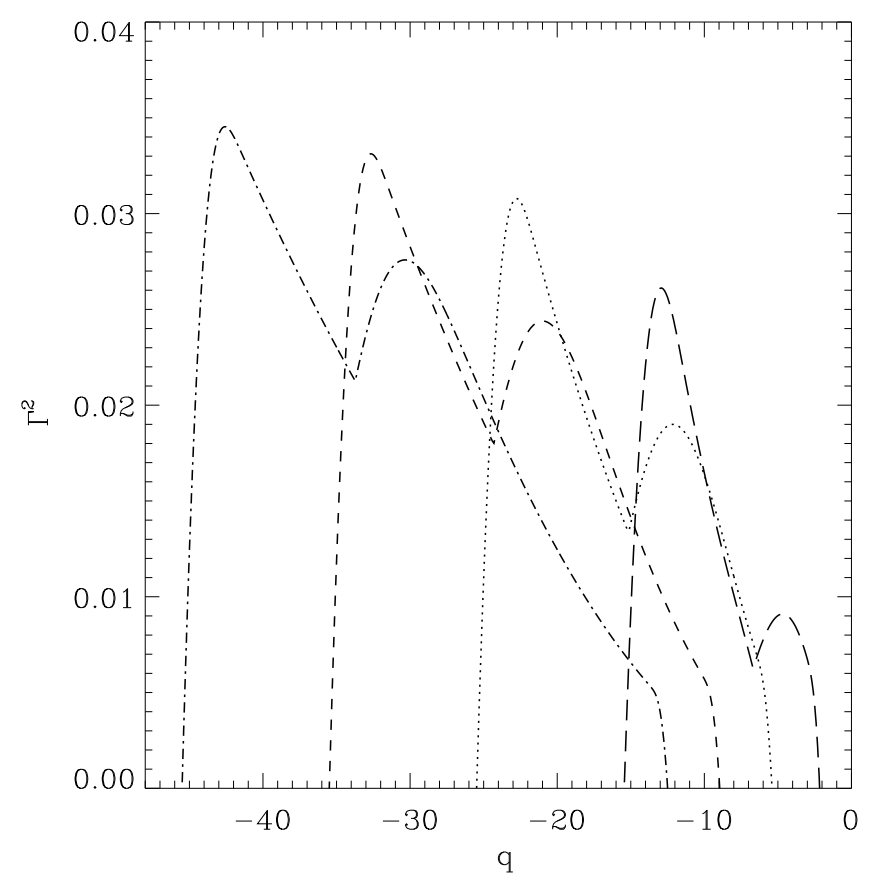

Fig. 7. The same as in Fig. 3 but for $\alpha=-0.5$.

In Fig. 8, the critical value of $\alpha_{\mathrm{c}}$ above which the system is unstable is plotted as a function of $\varepsilon$ for a range of vertical wavevectors relevant to stellar conditions. In fact, the local approximation in the axial direction applies if $\left|k_{z} s\right|>1$ that is equivalent $|q|>1$. On the other hand, to neglect gravity can be justified if $|q|>N / \omega_{B 0}$ (see Eq. (11)), which generally imposes a stronger restriction on $q$ since the ratio $N / \omega_{B 0}$ is typically $>1$ in stars. Therefore, in determining $\alpha_{\mathrm{c}}$, we assume that the allowed $|q|$ should be large enough and we choose $|q| \geq 10$, which corresponds to axial wavelengths shorter than $\lambda_{\mathrm{c}} / 10$. Since the 


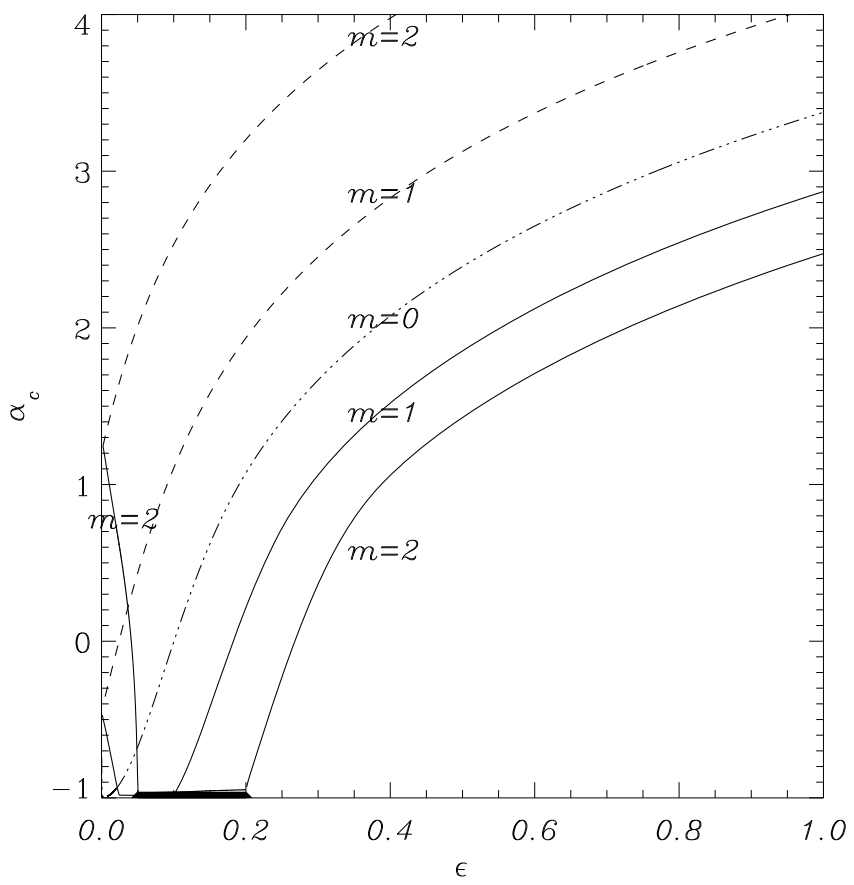

Fig. 8. The critical value of $\alpha$ that determines the onset of instability as a function of $\varepsilon$ for $|q|=10$. Solid and dashed curves correspond to $q=10$ and $q=-10$, respectively. The dash-and-dotted curve show critical $\alpha$ for $m=0$, which does not depend on the sign of $q$.

presence of an axial field breaks symmetry between positive and negative $q$, critical curves are different for positive and negative wavevectors. The region of $\alpha$ above the lines corresponds to configurations that are unstable for a given $m$. It turns out that true $\alpha_{\mathrm{c}}$, which determines instability, corresponds to perturbations with positive $m$ and negative $q$ as shown in Fig. 8 by solid lines. Critical $\alpha$ decreases with decreasing $\varepsilon$ everywhere except a region of small $\varepsilon$ where the dependence $\alpha_{\mathrm{c}}(\varepsilon)$ is very sharp. This particularly concerns the curve $m=0$, which goes up very sharply at $\varepsilon<0.01$ and reaches the value 1 at $\varepsilon=0$ in agreement with the result by Tayler (1973). However, the scale of Fig. 8 does not let us see this sharp behaviour. Unfortunately, our code does not allow to follow the behaviour of critical $\alpha$ when it approaches the value -1 because of the singular character of the last term on the left hand side of Eq. (15). The corresponding region is marked by crosses in Fig. 8. A more refined consideration is needed for this case which should perhaps include dissipation.

\section{Discussion}

We have considered the linear stability of magnetic configurations containing the toroidal and axial fields, assuming that the behavior of small perturbations is governed by equations of the non-dissipative incompressible magnetohydrodynamics. This approximation is justified if the magnetic field is subthermal and the Alfvén velocity is low compared to the sound speed. The stability of magnetic configurations is a key issue for understanding the properties of various astrophysical bodies such as peculiar A and B stars, magnetic white dwarfs and neutron stars. The magnetic instability can alter qualitatively the properties of configurations generated, for example, by dynamo in stars. Many dynamo models predict that the toroidal field should typically be stronger than the poloidal one, but such configurations can be unstable if the generated toroidal field does not decrease enough rapidly with $s$. The instability generates large- and small-scale motions that should alter the geometry of a generated magnetic field.

Even though the poloidal field is weaker than the toroidal one in a number of dynamo models, its effect on the stability properties cannot be neglected. This particularly concerns the behavior of the nonaxysimmetric perturbations considered in the present paper. If $B_{z}$ is relatively weak $\left(B_{z}<B_{\varphi}\right)$ then, typically, there exists a wide range of the azimuthal wave-numbers $m$ for which the instability may occur. For any given $m$, only perturbations within some particular range of the vertical wave-vectors $k_{z}$ can be unstable. The growth rate is maximal for perturbations with $k_{z}$ of about

$k_{z} \sim-\frac{m}{s} \frac{B_{\varphi}}{B_{z}}$

Equation (19) is approximately equivalent to the condition that the Alfvén frequency is vanishing somewhere within the cylindrical layer (see Eq. (17)). It should be noted that the ratio $B_{z} / B_{\varphi}$ can be rather low in stars, and the maximum growth rate at given $m$ corresponds to very short axial wavelengths,

$\lambda_{z} \sim 2 \pi \frac{s}{m} \frac{B_{z}}{B_{\varphi}}$

Taking, for example, $B_{z} / B_{\varphi} \sim 10^{-2}$ and assuming that $s$ is comparable to the stellar radius, $s \sim 10^{11} \mathrm{~cm}$, we find that the most rapidly growing modes should have the axial wavelength $\sim 6 \times 10^{9} / \mathrm{m} \mathrm{cm}$. From our results it follows that the maximum growth rate increases slowly with increasing $m$ and, therefore, perturbations with a very short azimuthal wavelength (very large $m$ ) should dominate the development of instability. For instance, the most rapidly growing mode with $m=100$ corresponds to the axial wavelength $\sim 6 \times 10^{7} \mathrm{~cm}$, which is very short compared to the radius. Therefore, the instability of magnetic configurations can often be determined by the modes with very large $m$ and an extremely short wavelength in the $z$-direction. This fact can cause problems in numerical modeling of the instability because simulations will require a very high resolution in the $\varphi$ and $z$-directions.

Depending on the profile of the toroidal field and the strength of the axial field, the instability can arise in two essentially different regimes. In the case of a weak axial field, $B_{\varphi 0} \gg B_{z}$, the value of $\alpha$ that distinguishes between the regimes is $\approx-1 / 2$. If $\alpha>-1 / 2$, then the instability grows on the Alfvén timescale determined by the toroidal field and is rather fast. In this case, the growth time is

$\tau \sim 0.1 \rho_{-4}^{1 / 2} s_{11} B_{\varphi 3}^{-1} \quad \mathrm{yr}$,

where $s_{11}=s / 10^{11} \mathrm{~cm}$. If $\alpha<-1 / 2$, then the growth time is given by same expression (18) but where $B_{\varphi 3}$ should be replaced by $B_{z 3}=B_{z} / 10^{3} \mathrm{G}$. Since $B_{\varphi} \gg B_{z}$, the instability is slower and grows on the timescale determined by the axial field in this case. The transition between two regimes occurs at larger $\alpha$ if the axial field increases.

It is fairly difficult to compare our results obtained for a simple model with the available numerical simulations, which usually use completely different basic magnetic configurations. For example, in calculations by Braithwaite $(2006,2007)$, the basic configuration was assumed to be either purely toroidal or purely poloidal, and the stability properties of such configurations differ qualitatively from those considered in this paper. Recently, Braithwaite \& Nordlund (2006) and Braithwaite (2008) have considered stability of the magnetic configuration with random 
initial fields. A vector potential was set up as a random field containing spatial scales up to a certain value. This random field was then multiplied by some screening function, so that the field strength in the atmosphere was negligible.

This initial configuration contains both the toroidal and poloidal fields but is very different from our simple model. Nevertheless, some features seem to be in common even for such different models. Braithwaite \& Nordlund (2006) and Braithwaite (2008) find that the instability can lead to different equilibrium configurations depending on the screening function. If the screening function for random fields decreases slowly or does not decrease at all, then the final equilibrium magnetic configuration is essentially non-axisymmetric. In contrast, an equilibrium configuration is closer to axisymmetry (but not axisymmetric) if the screening function decreases rapidly. This dependence on equilibrium configurations obtained in numerical calculations can reflect the regimes of "strong" and "weak" instabilities that correspond to different growth rates depending on the value of $\alpha$. In accordance with our analysis, the growth rate at given $\varepsilon$ is higher for higher $\alpha$ (compare, e.g., Figs. 3 and 7). Our parameter $\alpha$ mimics to some extent the screening parameter $p$ introduced by Braithwaite (2008) with decreasing $\alpha$ corresponding to increasing $p$. Therefore, we can expect from our analysis that non-aximetric instabilities should be more efficient for the screening function with $p=0$ than with $p=1$ and that the final configuration exhibits stronger departures from axisymmetry for smaller $p$. This conclusion seems to be in qualitative agreement with the results of Braithwaite (2008).

Our simple model does not take into account the stratification that can be important in many astrophysical applications. Basically, stratification provides a stabilizing influence if the temperature gradient is sub-adiabatic. However, this influence is small if perturbations have a relatively short wavelength in the axial direction, $\lambda<\lambda_{\mathrm{c}}$, such that inequality (11) is satisfied. Our results are related to this case. The case when $\lambda>\lambda_{\mathrm{c}}$ and stratification is important will be considered elsewhere.

Acknowledgements. This research project was supported by a Marie Curie Transfer of Knowledge Fellowship of the European Community's Sixth Framework Program under contract number MTKD-CT-002995. V.U. also thanks INAF-Ossevatorio Astrofisico di Catania for hospitality.

\section{References}

Acheson, D. 1973, J. Fluid Mech., 61, 609

Bonanno, A., \& Urpin, V. 2008, A\&A, 477, 35

Borra, E., Landstreet, J., \& Mestel, L. 1982, ARA\&A, 20, 191

Braithwaite, J. 2006, A\&A, 453, 687

Braithwaite, J. 2007, A\&A, 469, 275

Braithwaite, J. 2008 [arXiv: astro-ph/0801.4562]

Braithwaite, J., \& Nordlund, A. 2006, A\&A, 450, 1077

Braithwaite, J., \& Spruit, H. 2006, A\&A, 450, 1097

Dubrulle, B., \& Knobloch, E. 1993, A\&A, 274, 667

Kitchatinov, L. L., \& Rüdiger, G. 2007 [arXiv: astro-ph/0701847]

Knobloch, E. 1992. MNRAS, 255, 25

Markey, P., \& Tayler, R. 1973, MNRAS, 163, 77

Markey, P., \& Tayler, R. 1974, MNRAS, 168, 505

Mestel, L. 1999. Stellar Magnetism (Oxford: Clarendon)

Papaloizou, J., \& Terquem, C. 1997, MNRAS, 287, 771

Prendergast, K. 1956. ApJ, 123, 498

Press, W. H., Teukolsky, S. A., Vetterling, W. T., \& Flannery, B. P. 1992, Numerical Recipies in FORTRAN, The art of scientific computing (Cambridge: UP)

Spruit, H. 1999, A\&A, 349, 189

Terquem, C., \& Papaloizou, J. 1996, MNRAS, 279, 767

Tayler, R. J. 1957, Proc. Phys. Soc. B, 70, 31

Tayler, R. J. 1973, MNRAS, 161, 365

Tayler, R. J. 1980, MNRAS, 191, 151

Van Assche, W., Tayler, R. J., \& Goosens, M. 1982, A\&A, 1966

Wright, G. A. E. 1973, MNRAS, 162, 339 